\title{
The potential impact of the COVID-19 pandemic on HIV, tuberculosis, and malaria control in Africa: a systematic review of modelling studies and population surveys
}

Floriano Amimo ( $\nabla$ florianoamimo@gmail.com )

The University of Tokyo, Eduardo Mondlane University https://orcid.org/0000-0003-1460-9522

Ben Lambert

Imperial College London

Anthony Magit

University of California San Diego

Masahiro Hashizume

The University of Tokyo

\section{Systematic Review}

Keywords: COVID-19, SARS-CoV-2, HIV, tuberculosis, malaria, health policy, health system, disease burden, infectious diseases modelling, Africa

Posted Date: November 6th, 2020

DOI: https://doi.org/10.21203/rs.3.rs-103235/v1

License: (9) This work is licensed under a Creative Commons Attribution 4.0 International License.

Read Full License 


\section{Abstract}

Background We reviewed current evidence on the impact of COVID-19 on HIV, tuberculosis, and malaria across Africa.

Methods We searched medical databases up to 15 September 2020 for epidemiological studies that investigated the impact of COVID-19 on HIV, tuberculosis, and malaria in terms of morbidity, mortality, or healthcare accessibility, covering African countries. We consulted global health and government sources for population surveys providing relevant primary data. We employed meta-study to assess evidence. This study is registered with PROSPERO, number CRD42020209265.

Results Of the 128 records screened, 14 epidemiological studies were included in the analysis. We identified 5 population surveys. The largest effect of COVID-19 related disruption of HIV, TB, and malaria services is projected to result from interruption of ART, decline in TB case detection, and interruption of insecticide treated bed nets distribution campaigns, respectively. However, most modelling studies substantially underestimated the potential implications of COVID-19 on HIV, TB, and malaria in Africa and focused on the disruption of healthcare resulting from reduction in the capacity of healthcare provider. Reduced capacity of patients to use health services and adhere to treatment may also contribute to poor outcomes even when health systems capabilities are restored or unaffected. Moreover, these models do not account for any potential interaction of HIV, TB, and malaria with COVID-19, although empirical evidence shows that coinfection between HIV and TB with COVID-19 is significantly associated with increased mortality risk in Africa.

Conclusions Current evidence is largely speculative. Innovative COVID-19 resilient solutions calibrated to local vulnerabilities are vital to ensure continuity of care and prevent the emergence of further potentially larger crises.

\section{Background}

In the face of a rapidly growing coronavirus disease 2019 (COVID-19) with a high transmissibility and no cure or vaccine, many governments around the world adopted sweeping and unprecedented nonpharmaceutical measures. These measures were adopted largely without robust prior evidence regarding their population-level effectiveness and societal implications of their implementation. Most studies conducted after COVID-19 containment and mitigation measures were already implemented have indicated that these measures might have reduced the spread of the pandemic and averted millions of potential COVID-19 deaths. ${ }^{1-7}$ However, these studies have covered mostly North America, Europe, and parts of Asia - which have so far been heavily affected by the pandemic compared to most African countries. Therefore, the effectiveness and sustainability of these measures at national or subnational level across Africa remains debatable. 
Several factors have been associated with the reduced incidence, severity, and fatality of severe acute respiratory syndrome coronavirus 2 (SARS-CoV-2) infection observed across Africa, despite the risks associated with economic and health systems vulnerabilities, including limited testing and surveillance capabilities. ${ }^{8-20}$ Nevertheless, most African countries have continued enforcing these measures, or at least part of them, typically longer and/or more stringently than many advanced economies. Serious concerns have been raised in the scientific community regarding the potentially detrimental public health consequences of COVID-19 control measures on non-COVID-19 diseases, in particular human immunodeficiency virus (HIV) /acquired immunodeficiency syndrome (AIDS), tuberculosis (TB), and malaria. These are highly endemic across Africa, remain major causes of death and suffering on the continent, and some of them are more fatal than COVID-19. Reviews of empirical evidence from the 20142016 Ebola outbreak in west Africa have indicated that the indirect health impact of the outbreak was more severe than the disease itself. ${ }^{21-23}$ The usually under-funded and ill-equipped health systems in many countries across Africa compound the risks. ${ }^{24}$ These might be worsened by the disruption of the international system affecting the supply of essential medical products, as well as reduced capacity of patients to support direct and indirect medical costs, reduced inclination of patients (including those presenting symptoms overlapping with those of the new pandemic) to seek care at a formal health facility due to COVID-19 related fears, and clinical parallels and potential interactions between COVID-19 and these diseases. ${ }^{25}$

Here, for the first time, we systematically review current evidence from studies that employed mathematical models and population surveys to quantify the potential public health consequences of COVID-19 on HIV, TB, and malaria across Africa. Although our focus is on studies that generated quantitative data, we use evidence from studies providing relevant insights to draw comprehensive policy recommendations to mitigate the emerging risks across the continent and improve preparedness of African countries to future crises.

\section{Methods}

\section{Search strategy and selection criteria}

We searched LitCovid, Scopus, MEDLINE (OVID), CINAHL (EBSCO), Web of Science Core Collection, African Index Medicus, Cochrane Library, WHO databases, preprint servers, and Google scholar for epidemiological studies. We identified population surveys by consulting websites of Global Health organizations and government institutions. Using our pool of potentially eligible studies we conducted citation backtracking to identify additional records of epidemiological studies and population surveys. The search for evidence was done from 20 August 2020 to 15 September 2020 , covering records with publication year 2019-2020. Records were collated and reviewed using Endnote X8. (Table 1). 
We assessed the eligibility of our records in two instances. In the first instance, titles and abstracts were assessed for eligibility, and in the second instance full text of records deemed eligible in the first instance were reviewed. Eligibility assessment was done by $F A$ and validated independently by $B L, A M, M H$. We included studies measuring the potential/observed effects of COVID-19 on HIV, TB, or malaria in human populations; involving at least one Africa country; reporting results on relevant outcomes from original research; published in a peer reviewed journal or deposited in WHO database or a preprint server (for epidemiological studies). We excluded studies that did not generate quantitative data on relevant outcomes; whose full text is not available; published in a language other than English; not reporting data sources (for epidemiological studies), the analytical strategy employed (for epidemiological studies), and the key assumptions underpinning the validity of the results (for modelling studies). (Table 2).

Table 1: Search algorithm applied to medical databases to retrieve evidence pertaining the effects of COVID-19 on HIV, tuberculosis, and malaria. The algorithm was applied to LitCovid, Scopus, MEDLINE (OVID), CINAHL (EBSCO), Web of Science Core Collection, African Index Medicus, and Cochrane Library. Evidence gathering was done from August 20 to September 15, 2020.

\begin{tabular}{|c|c|}
\hline Set & Terms \\
\hline 1 & “COVID-19" or "SARS-CoV-2" \\
\hline 2 & "HIV" or "AIDS" or "tuberculosis" or "malaria" \\
\hline 3 & $\begin{array}{l}\text { "service" or "care" or "program" or "control" or "drug" or "treatment" or "therapy" or "prevention" } \\
\text { or "prophylaxis" or "hospital" or "testing" or "counselling" }\end{array}$ \\
\hline 4 & "access" or "cost" or "quality" or "safety" or "funding" \\
\hline 5 & $\begin{array}{l}\text { "prevalence" or "incidence" or "deaths" or "burden" or "vulnerability" or "severity" or } \\
\text { "hospitalization" }\end{array}$ \\
\hline 7 & "Africa" or "low-income" or "middle-income" \\
\hline 8 & 1 and $(2$ and $((3$ and 4$)$ or 5$))$ and 7 \\
\hline
\end{tabular}

Table 2: Eligibility criteria used for the inclusion and exclusion of studies identified through comprehensive search in medical databases. Inclusion criteria 4 are applicable for epidemiological studies. Exclusion criteria 4 are applicable for epidemiological studies as follows: not reporting data 
sources and analytical strategy employed (epidemiological studies) and not reporting the key assumptions underpinning the validity of the results (modelling studies).

\begin{tabular}{|c|c|}
\hline Inclusion Criteria & Exclusion Criteria \\
\hline We included studies: & We excluded studies: \\
\hline $\begin{array}{l}\text { 1. covering the potential effects of COVID-19 } \\
\text { on HIV, tuberculosis, or malaria in human } \\
\text { populations }\end{array}$ & $\begin{array}{l}\text { 1. that did no generate quantitative data on relevant } \\
\text { outcomes }\end{array}$ \\
\hline & 2. whose full text is not available \\
\hline & 3. published in a language other than English \\
\hline from original research & \multirow{2}{*}{$\begin{array}{l}\text { 4. not reporting data sources, the analytical strategy } \\
\text { employed, and the key assumptions underpinning } \\
\text { the validity of the results }\end{array}$} \\
\hline $\begin{array}{l}\text { 4. published in a peer reviewed journal or } \\
\text { deposited in World Health Organization } \\
\text { database or a preprint server }\end{array}$ & \\
\hline
\end{tabular}

\section{Data extraction and quality assessment}

For each record that passed title-abstract eligibility assessment, we extracted data on study objective, geographical coverage, target populations, study outcomes, data sources, sample size, analytical strategy, model assumptions, main findings, and study limitations. From studies that were deemed eligible at full-text eligibility assessment, we extracted quantitative data on the potential/observed effects of [COVID-19 or SARS-CoV-2] on [service, care, program, control, drug, treatment, therapy, prevention, prophylaxis, hospital, testing, or counselling] or [access, cost, quality, safety, funding, prevalence, incidence, deaths, burden, disability adjusted life years lost, vulnerability, severity, or hospitalization] in relation to [HIV/AIDS, tuberculosis, or malaria] in any African country. For any of these study outcomes, we extracted data on the relative/absolute increase/reduction associated with exposure to SARS-CoV-2 (infection), COVID-19 (disease), or COVID-19 pandemic (disruption). We considered studies comparing the predicted/observed impact of any of these exposures on HIV, tuberculosis, or malaria outcomes versus a counterfactual/observed scenario/period of no SARS-CoV-2 (infection), COVID-19 (disease), or COVID-19 pandemic (disruption). Data were extracted by FA and validated independently by $B L, A M, M H$. 
This study complies with the guidelines outlined in Preferred Reporting Items for Systematic reviews and Meta-Analyses (PRISMA). (Supplement 1.1). The quality of each potentially eligible study was assessed following the guidelines outlined by Cochrane tool for assessing risk of bias in a non-randomized study, when applicable. We also assessed modelling studies based on their compliance with the Guidelines for Accurate and Transparent Health Estimates Reporting (GATHER). We used details on study outcomes, sample size, data sources, analytical strategy, model assumptions, and study limitations, extracted from each study, to assess quality. (Supplement 2.1). This study is registered with PROSPERO, number CRD42020209265.

\section{Evidence synthesis}

Due to large heterogeneity across studies, we limited our analysis to qualitative evidence synthesis using meta-study. This is a multi-faceted approach, comprising meta-data-analysis (analysis of findings), metamethod (analysis of methods), and meta-theory (analysis of theory). Evidence synthesis was conducted when at least four eligible studies were identified. (Supplements 1.1-2.2).

\section{Results}

We identified a total of 190 records through database searches and citations backtracking. After removing duplicates, 128 unique records were subjected to title-abstract eligibility assessment, which resulted in the exclusion of 99 records. Therefore, 29 full-text articles were reviewed, of which 15 were excluded. As a result, 14 epidemiological studies were included in the data extraction. After consulting additional sources, we identified 5 relevant population surveys, which were added to the evidence pool. (Figure 1, Supplement 2.1). Among the included epidemiological studies, 7 measured the impact of COVID-19 on HIV (covering Uganda, South Africa, Malawi, Zimbabwe, Uganda, sub-Saharan Africa (SSA), and low- and middle-income countries (LMIC)), ${ }^{26-31} 5$ measured the impact of COVID-19 on TB (covering China, India, Kenya, Ukraine, Sierra Leone, South Africa, Zimbabwe, the world), ${ }^{27,31-34}$ and 6 measured the impact of COVID-19 on malaria (covering Sierra Leone, Uganda, SSA, and LMIC). ${ }^{26,27,34-37}$ The included population surveys were conducted by (and covered) FinMark Trust (Kenya, Nigeria and South Africa), ${ }^{38}$ Human Sciences Research Council (South Africa), ${ }^{39}$ International AIDS Society (Zimbabwe), ${ }^{40}$ National Institute for Communicable Diseases (South Africa), ${ }^{41}$ and the Global Fund (Latin America, the Caribbean, and high-burden countries in Africa). ${ }^{42}$ (Supplements 2.2-2.3). 
Despite large variability due to differences in model assumptions and disruption scenarios across studies, most predictions suggest that the largest impact of COVID-19 related disruption of essential health services on HIV burden across SSA might result from interruption of antiretroviral therapy (ART) among people living with HIV/AIDS (PLWHA) who are already under ART. A 6-month interruption of ART for $50 \%$ of PLWHA on ART is projected to result in an excess HIV-related deaths over 1 year (from 1 April 2020 ) of 296,000 (median across models; range $229,023-420,000$ ) (95\% of which aged $<65$ years) in the region, compared with the counterfactual scenario of no COVID-19 related disruptions. ${ }^{28}$ The effect is projected to be larger in countries with very high HIV prevalence, where a forced interruption of ART under a no action or suppression-lift COVID-19 scenario could result in $10 \%$ increase in the number of HIV deaths over a 5-year period. ${ }^{27}$ The largest impact of disruption of ART on mother-to-child transmission (MTCT) of HIV is projected to be felt in areas with high coverage of prevention of mother-to-child transmission (PMTCT) programs, such as Malawi, Mozambique, Uganda, and Zimbabwe. In these countries, a suspension of 3-month of PMTCT could result in a relative increase in MTCT of HIV over 1 year (from 1 April 2020) of 1.81, 1.41, 1.70, and 1.53, respectively, whereas across SSA the relative increase would be of 1.64 under a 6-month interruption of ART for $50 \%$ of PLWHA on ART. ${ }^{28}$ The impact on HIV incidence and mortality of COVID-19 related disruption of other HIV services is projected to be relatively lower compared to interruption of ART in the short-term, with the median predicted relative change over 1-year in HIV incidence and mortality ranges from 1.00 to 1.17 and from 0.99 to 1.38 , respectively. ${ }^{28}$

A study ${ }^{31}$ using population cohort data from Western Cape Province, South Africa, has indicated that coinfection of COVID-19 and HIV is associated with increased mortality. Comparing HIV positive with HIV negative patients among COVID-19 cases, the study found that those that are HIV-positive have an agesex adjusted mortality rate 2.39 (1.96-2.86) times greater that those who are HIV negative. At populationlevel, 8.5\% (6.1-11.1) of deaths among COVID-19 patients is attributable to HIV infection. Likewise, when those with current TB were compared with those without current TB among COVID-19 patients, those with current TB had a hazard of death $2.70(1.81-4.04)$ times greater. When the comparison was done between those with previous TB vs those without previous TB among COVID-19 patients, the study found that those with previous TB have hazard of death $1.51(1.18-1.93)$ times greater.

Nevertheless, the major public health consequence of COVID-19 related disruption on TB burden across Africa is projected to result from reduction in case detection. Overall, $20 \%$ excess increase in the number of TB deaths over 5-year are projected across LMIC, under a suppression scenario in country setting 1 (very high burden setting with 520 TB incidence per 100,000 population in 2018) compared to the counterfactual scenario of no COVID-19 related disruptions. ${ }^{27} \mathrm{~A}$ global average decline of $25 \%$ and $50 \%$ in TB case detection over a period of 3 months compared to levels before the pandemic is projected to result in $13 \%$ and $26 \%$ increase in TB deaths in 2020 globally, respectively. ${ }^{33}$ Across the WHO African 
region which accounts for over $25 \%$ of global TB deaths, these reductions in case detection would result in $>47,500(14,000-101,500)$ and $>95,000(39,000-176,500)$ excess TB deaths in 2020 , respectively.

TB programs across high burden countries in Africa have been equally affected by the aforementioned disruptions in in-country supply chain systems (distribution), in-country supply chain systems (warehouse), and HIV and/or TB laboratory services. ${ }^{42}$ The COVID-19 pandemic has also compromised health service delivery for TB programs, with $75 \%$ countries reporting moderate, high, or very high disruption. ${ }^{42}$ In South Africa, weekly TB Xpert testing volumes during the period under COVID-19 level 5 restrictions and weekly number of TB Xpert positive tests during the period under COVID-19 level 5 restrictions declined by $48 \%$ and $33 \%$, respectively, compared with the period before COVID-19 lockdown. ${ }^{41}$ The reduction in access to, and increased cost of, medicines during the lockdown reported in population-based surveys conducted in Kenya, Nigeria and South Africa compound the deleterious effects of COVID-19 related disruptions on TB control efforts. ${ }^{38,39}$ In Zimbabwe, 23\% of PLWHA indicated lack of access to TB treatment during the COVID-19 pandemic. ${ }^{40}$

The major impact of COVID-19 on malaria control is projected to result from disruption of insecticide treated bed nets (ITN) distribution and of malaria case management (MCM). In the short term, those countries that have a campaign scheduled for 2020 will be affected the most. However, indoor residual spraying (IRS) and seasonal malaria chemoprevention (SMC) are also expected to experience disruption, at varying levels across the continent. In the best scenario (World Health Organization (WHO) scenario 1 (WS1)), with no ITN campaigns, and continuous ITN distribution reduced by $25 \%$, about $230,527,960$, 197,898,185, and 32,629,775 malaria cases are predicted for the period between April-December 2020 in all countries, countries with ITN campaigns scheduled in 2020, and countries without ITN campaigns scheduled in 2020, respectively. This would result in $411,684,349,194$, and 62,489 malaria deaths in these countries over the same period, respectively. However, in the worst scenario (WS9), with no ITN campaigns, and both continuous ITN distribution and access to effective antimalarial treatment reduced by $75 \%$, then $261,582832,224,886,788$, and $36,696,044$ malaria cases would occur in these countries over the same period, respectively. This would represent $768,588,656,251$, and 112,337 malaria deaths in these countries over the same period, respectively. ${ }^{37}$

However, the impact of COVID-19 on malaria control would be influenced by the type of COVID-19 responses adopted across the continent. The largest excess deaths would be observed if ITN, SMC, MCM are all interrupted. In this case, 253,000 (149,000-357,000), 481,000 (277,000-686,000), 696,000 $(413,000-978,000)$, and $484,000(278,000-690,000)$ excess malaria deaths would occur under the COVID-19 scenario of unmitigated, mitigation, suppression, and suppression lift, compared with the 
counterfactual scenario of no COVID-19 related disruption, respectively. The lowest impact would be observed if ITN is normal, but SMC and MCM are reduced, in which case 26,000 (15,000-38,000), $112,000(61,000-163,000), 200,000(115,000-285,000), 112(61,000-164,000)$ excess malaria deaths would occur under each COVID-19 scenario, respectively. However, if ITN and SMC are interrupted, but MCM is reduced, then 239,000 (141,000-337,000), 379,000 (221,000-537,000), 464,000 (278,000$651,000)$, and $380,000(222,000-539,000)$ excess malaria deaths would occur under each COVID-19 scenario, respectively. Whereas if ITN is interrupted, but SMC and MCM are reduced, then 220,000 $(128,000-311,000), 357,000(207,000-507,000), 495,000(296,000-693,000)$, and 358,000 (208,000$509,000)$ excess malaria deaths would occur under each COVID-19 scenario, respectively. ${ }^{36}$

Data from population-based studies show that the ongoing COVID-19 pandemic has negatively impacted the access to, and cost of, health services relevant for HIV, TB, and malaria control on the continent. Across high-burden countries in Africa (as well as Latin America, the Caribbean), $80 \%, 58 \%$, and $66 \%$ countries have reported moderate, high, or very high disruption of health service delivery for HIV, TB, and malaria programs, respectively. ${ }^{42}$ In South Africa, a cohort study has shown that women had an odds ratio of $2.36(1.73-3.16)$ to miss a medical visit for pre-exposure prophylaxis in pregnant and postpartum women during the lockdown compared to before lockdown; ${ }^{43}$ study visits were scheduled the same time as antenatal or postnatal visits, therefore this figure also reflects the increase in the propensity of women to miss antenatal and postnatal visits at a primary care clinic because of COVID-19 lockdown, thus affecting HIV, TB and malaria services that are deployed during antenatal and/or postnatal care. Additionally, $13.2 \%$ and $13-25 \%$ general populations and populations living in informal settlements in the country have indicated that chronic medication was inaccessible during the lockdown, respectively. ${ }^{39}$ In Nigeria and Kenya, $10 \%$ and $30 \%$ of $18+$ years old general population reported having changed the way they access medical care for chronic conditions because of COVID-19 related disruptions, respectively. ${ }^{38}$ In Zimbabwe, cost of medicines is the main challenge in getting antiretroviral drugs (ARV) in time during the COVID-19 pandemic for $23 \%$ of PLWHA. ${ }^{40}$ In Kenya and Nigeria, $42 \%$ and $50 \%$ of $18+$ years old general population report that medicines are more expensive since 1 March 2020 than before, respectively. ${ }^{38}$ Nevertheless, the effect of COVID-19 seems to vary across and within countries, potentially affecting less the capacity of health facilities to delivery HIV services across rural Africa. For example, data from (rural) northern KwaZulu-Natal, South Africa, shows that even though daily HIV testing, antiretroviral therapy initiation, antiretroviral therapy continuation, or pharmacy pick-up per week during post-lockdown period declined by 1.5 (95\% uncertainty interval, -3.4-0.3) compared to pre-lockdown period, HIV clinic visits per day increased by 8.4 (2.4-14.4) immediately after the lockdown implementation compared to pre-lockdown period. ${ }^{30}$ In contrast, in Zimbabwe, a much larger segment of PLWHA reported that they did not get ARV refills because of COVID-19 related disruptions. Among these, $60 \%$ do not know where to go to get their HIV medications because of closure of their usual pharmacy in the context of COVID-19 restrictions and 31\% do not know where to go to get their HIV medications because they are not in the part of the country where they usually access HIV medications. Furthermore, 
$43 \%$ of PLWHA lack access to condoms during the COVID-19 pandemic. ${ }^{40}$ In addition to health services delivery and access to medicines, the COVID-19 pandemic has also disrupted the supply systems that are needed to ensure the medical products necessary for HIV programs. Across high-burden countries, $24 \%$, $15 \%$, and $58 \%$ countries have reported moderate, high, or very high disruption in: in-country supply chain systems (distribution), in-country supply chain systems (warehouse), and HIV and/or TB laboratory services, respectively. ${ }^{42}$

Table 3: COVID-19 resilient service delivery strategies and economic solutions to ensure adherence of HIV and TB patients to treatment and improve malaria clinical outcomes. COVID-19 denotes coronavirus disease 2019. HIV denotes human immunodeficiency virus. IPTi denotes intermittent preventive treatment in infancy. IPTp denotes Intermittent preventive treatment in pregnancy. ITN denotes insecticide-treated nets. MCM denotes malaria case management. SMC denotes seasonal malaria chemoprophylaxis. TB denotes tuberculosis.

Strategies to ensure $\quad$ Target populations and implementation strategies
adherence to
treatment and improve
outcomes

Multi-month dispensing of medication coupled To ensure continuity of care and access to medicines among HIV and TB with instructions for safe storage at home

Mobile clinics patients

(HIV and TB control and prevention). To distribute anti-malarial drugs for
preventive (SMC, IPTp, and IPTi) and curative treatments (MCM), and for
malaria testing and ITN distribution (malaria control)

Virtual care and digital health technology Conditional cash transfer

Economic support
To implement adherence psychosocial support and group-based and community-centric service delivery models for HIV and TB patients

To promote adherence to treatment among HIV and TB patients and incentivize TB screening, through banks accounts or mobile money

To promote adherence to treatment among HIV and TB patients and improve malaria clinical outcomes. These might be in the forms of nutritional/transport allowance, income generation schemes, and/or microfinance loans (e.g., health saving plans, emergency health loans)

\section{Discussion}


This is the first systematic review of the implications of COVID-19 on HIV, TB, and malaria. Drawing on quantitative data on projected burden of these diseases and COVID-19 under various disruption and response scenarios generated by modelling studies and primary data derived from population surveys that covered African countries, our study unifies current evidence and provides comprehensive policy recommendations, to facilitate translation of the extensive evidence generated so far into effective policies across the continent.

Three modelling studies ${ }^{27-29}$ assumed a $10 \%$ reduction in the risk of acquiring HIV due to a reduction in the rate of formation of new sexual partnerships. Although COVID-19 related social distancing measures might in the short-term produce such positive effect, thus reducing casual and transactional sex, in the mid- and long-term the opposite effect might be observed. The COVID-19 crisis is projected to almost double the number of people with acute hunger by the end of $2020 .{ }^{44}$ Moreover, recent reports show that as a result of COVID-19 related measures, an additional $9.1 \%$ of the population across SSA are estimated to have fallen into extreme poverty, with $30 \%$ of the population across the continent projected to lose their resilience capacity to future shocks under an eight-week lockdown scenario. ${ }^{45}$ Further estimates show that without social and economic mitigation measures, 80 million more people are expected to fall into extreme poverty, representing a $23 \%$ increase. ${ }^{46}$ On the other hand, global recession following COVID-19 coupled with plummeting oil prices and a lowered global demand for African non-oil products means that many governments across the continent will have reduced capacity to extend the public services. ${ }^{47,48}$ Empirical evidence has shown that food insecurity is associated with increased sexual risk through transactional sex and inability to negotiate safer sex. ${ }^{49,50}$ Hunger and food insecurity are also barriers for ART initiation and adherence, thus further worsening clinical outcomes and increasing HIV transmissibility. Reduced access to condoms, as reported by $43 \%$ of PLWHA surveyed in Zimbabwe, ${ }^{40}$ compounds the risks of increased HIV transmission because of COVID-19 disruptions. Moreover, these models ${ }^{27-29}$ do not account for the effects of potential interaction of HIV, TB, malaria with COVID-19. However, a study based on population cohort in Western Cape, South Africa, has shown that SARS-CoV-2 and HIV coinfection is associated with hazard of COVID-19 death in the general population 2.14 (1.702.70) times greater in HIV positive patients compared with HIV negative patients. ${ }^{31}$ The same study also indicated an association between SARS-CoV-2 and Mycobacterium tuberculosis coinfection (current or previous TB) and an increased hazard of death; the quantity (comparable) and direction of such link has been confirmed elsewhere (relative risk: $2.17(1.40-3.37)$ ). ${ }^{51}$ The interaction between COVID-19 and HIV has also been reported in a prospective cohort study involving HIV positive adults in Madrid, Spain, indicating that younger PLWHA are more prone to COVID-19 and have a higher age-adjusted mortality (mortality among those aged $50-59$ years: $8 \%$ vs $4 \%$ ) than their counterpart with the same age in the general population. ${ }^{52}$ Empirical evidence from the United States also supports the link, although the study highlighted the role of CD4 count $<200$ cells $/ \mathrm{mm}^{3}$ on hospitalization and severe outcome risks, regardless of viral supression. ${ }^{53}$ This means that the quantities generated in these studies ${ }^{27-29}$ substantially underestimate the potential impact of COVID-19 on HIV and TB burden. 
Empirical evidence on mechanisms underlying potential impact of COVID-19 on TB is still limited. However, late reactivation of TB (because of the detrimental effects of COVID-19 on immune system, respiratory system, and national economies) has been indicated as a possible path by which COVID-19 might affect TB clinical outcomes. ${ }^{54}$ The risk of household transmission might be exacerbated by prolonged household contact. ${ }^{55}$ The consequences of such increased household transmission due to intense household exposure might not be observed in the short-term because of long incubation period of TB. Reinforcing strategies to mitigate the risk of household transmission of TB is therefore vital in the context of the ongoing crisis. ${ }^{54} \mathrm{~A}$ recently validated tool could be used to predict the risk of TB among contacts of patients with TB to guide the implementation of these mitigation strategies. ${ }^{56}$

Furthermore, reduced case detection, as reported in South Africa under COVID-19 level 5 restrictions compared with before the restrictions, means that TB prevalence is expected to increase following the current crisis. The decline in case detection reported in South Africa $(48 \%)^{41}$ is similar to the decline assumed by Glaziou et al. (50\%). ${ }^{33} \mathrm{~A}$ decline in TB case detection comparable to that reported in South Africa is likely to have occurred in other countries across the continent. Therefore, the $>95,000(39,000-$ 176,500) excess TB deaths estimated for 2020 in the WHO African region likely approach the actual TB burden attributable to decline in TB case detection in the region in the short-term (which we computed by using global estimates of excess TB deaths and the global share of TB deaths across WHO African region (>25\%)), although the effects of COVID-19 disruptions are expected to last several years. A study conducted in the European Union during 2008-2011 assessing the relationship between TB control and economic recession indicated that a decline of case detection of $5 \%$ was followed by a $3 \%$ increase in TB prevalence and TB deaths for more than a decade after the recession. ${ }^{57}$ Non-adherence to treatment further affects HIV and TB burden by facilitating the emergence of drug-resistant pathogens. The current crisis because of COVID-19 creates all the conditions that have been indicated in previous studies as being associated with HIV and TB treatment non-adherence and lost to follow across the continent. $^{25,49,58-67}$

The cost-effectiveness of various innovative solutions for service delivery is well documented across the continent, ${ }^{54,68-82}$ warranting their utility to mitigate the risks posed by COVID-19 (Table 3); however, some constraints have been reported. In relation to mobile clinics, these include logistical challenges as well as spatial and structural constraints. ${ }^{83}$ For example, home delivery of ART might result in inadvertent disclosure of HIV status to family member, thus compromising acceptability of mobile clinics among PLWHA. Also, patients might change address and/or telephone number, thus making it difficult to reach them. Therefore, efforts to ensure consent, confidentiality, and privacy of patients as well as full engagement with the community and culturally informed recruitment of HCW are pivotal for their 
successful implementation. Digital solutions might exacerbate health inequalities, particularly in relation to people living in extreme poverty, the elderly, rural populations, and other vulnerable segments of society. For these populations, digital solutions need to be accompanied with sustainable and effective economic and technological solutions. Overall, integrated service delivery is vital to ensure costeffectiveness. ${ }^{84}$

To minimize the detrimental effects of COVID-19 on malaria it is vital to ensure continuity of ITN distribution, SMC, MCM, and other core malaria services. However, the already fragile capacity of patients to access health facilities or to purchase medicines is expected to be further compromised in the current crisis. Moreover, due to overlap of clinical features between malaria and COVID-19, medical care avoidance behavior is expected to increase in the current crisis, with patient going to hospital only in the worst-case scenario, thus resulting in poor clinical outcomes. Fear of acquiring SARS-CoV-2 infection and increased police violence could also reduce inclination of malaria patients to seek care at a formal health facility.

COVID-19 related disruptions have also compromised intermittent preventive treatment with sulfadoxinepyrimethamine (SP) in pregnancy (IPTp) and in infancy (IPTi) across Africa. Increased propensity of women to miss antenatal and postnatal medical visits, as reported in South Africa, ${ }^{43}$ reduces the number of doses of IPTp that women are able to receive. The WHO recommends at least three doses of SP to all pregnant women during antenatal care in the second trimester, each dose given at least a month apart; therefore, COVID-19 disruptions will impact negatively the effectiveness of IPTp, which is already compromised in Eastern Africa and parts of Central Africa in the context of declining drug efficacy driven by increasing levels of high-levels resistance to $\mathrm{SP}^{85} \mathrm{IPTi}$, which the WHO recommends to be given three times during the first year of life at 10 weeks, 14 weeks, and 9 months of age through immunization services, has also been negatively impacted by the COVID-19 disruptions, further compromising its already reduced effectiveness across the continent. ${ }^{85}$ A study conducted in Sierra Leone has found that IPTi1, IPTi2, and IPTi3 declined significantly by $69.4 \%, 65.9 \%$, and $51.1 \%$ during the COVID-19 lockdown compared to similar period in 2019 , respectively. ${ }^{34}$

Furthermore, empirical evidence shows that in water-based communities across Eastern Africa and in parts of Central and Western Africa, the use of free malaria bed nets for fishing is widespread. Previous work has indicated that mosquito net fishing is associated positively with poverty and food insecurity, with an increasing temporal trend. ${ }^{86-88}$ Therefore, the propensity of mosquito net fishing among waterbased communities across the continent will increase, thus undermining the effectiveness of ITN distribution campaigns. 
Overall, most of the modelling studies reviewed here consider only disruption resulting from reduced capacity of healthcare provider. Therefore, these studies substantially underestimate the severity and ramifications of potential impact of the COVID-19 pandemic on HIV, TB, and malaria. To effectively respond to COVID-19, programs to control these diseases need to be accompanied with sustainable economic solutions and COVID-19 resilient service delivery strategies (Table 3). Where these are already being implemented, ensuring their continuity, effectiveness, and sustainability is paramount.

Assessment of the relative weight of excess HIV, TB, and malaria burden compared to the new pandemic is complicated by differences across studies in model assumptions, underlying data, the number of projected COVID-19 deaths, and heterogeneity in COVID-19 control strategies across space-time. Nevertheless, the combined excess HIV, TB, and malaria deaths due to COVID-19 disruptions is significantly larger than the direct burden of a counterfactual unmitigated COVID-19 in most of the continent.

For HIV, under COVID-19 deaths projections based on Cabore et $\mathrm{al}^{9}{ }^{9}$ Walker et al, ${ }^{8}$ or Pearson et al, ${ }^{89}$ a 9month interruption of ART for 6,47 , or $90 \%$ of PLWHA on ART, respectively, could produce excess HIV deaths in 15+ years old over a 5-year period comparable to the projected number of COVID-19 deaths in South Africa, Malawi, Zimbabwe, and Uganda. ${ }^{29}$ Nevertheless, most studies use excess incidence and mortality to measure the impact of COVID-19 on HIV, TB, and malaria, even though these do not fully capture the impact of COVID-19 on HIV burden, compared to COVID-19 burden. DALYs lost should be used to better reflect demographic differences of populations at risk. Also, most studies focus in the number of cases, which may overestimate the implications of a new COVID-19 case compared with a new HIV case by implying that an individual being infected with SARS-CoV-2 is comparable with the counterfactual scenario of the same individual being infected with HIV in Africa. Although the severity of a SARS-CoV-2 infection in vulnerable segments of society has been well established, it is asymptomatic and self-limiting for most young populations, who constitute the vast majority across Africa. In contrast, the implications of a new HIV case are more detrimental to society across Africa because an HIV infection is severe in all demographic groups and tends to affect younger populations (who constitute the vast majority across Africa). For example, in Uganda, missed new diagnosis in 2020 based on 2019 6month average and a $20 \%$ loss to follow-up for 6-month is projected to produce an excess of 475,319 disability-adjusted life-years (DALYs) lost as a result of additional 41,757 PLWHA not initiating ART over 6-month. This figure, which does not account for COVID-19 related disruption of other HIV services and other pathways by which COVID-19 might impact HIV burden in the country, represents 70,219 excess DALYs lost compared to the DALYs projected to be lost in the country due to COVID-19 directly, under a $20 \%$ detectable incidence rate nationally. ${ }^{26}$ Furthermore, many African countries exhibit socioecological 
factors that have been indicated as being protective against COVID-19. In addition to younger populations, these protective factors include largely rural population, high bacillus Calmette-Guérin (BCG) vaccination index, high prevalence of latent tuberculosis infection (LTBI), proximity to the equator, among others. ${ }^{8-20}$

For TB, for every month of lockdown and every month of restoration, the excess TB deaths would be $1,700(1,200-2,260)$ and $1,070(753-1,380)$ in Kenya, respectively. ${ }^{32}$ In comparison, the WHO regional office for Africa projects 3,340 COVID-19 deaths in Kenya over 52 weeks of widespread community transmission of SARS-CoV-2. ${ }^{9}$ Although data from most of the continent is still limited, these data from Kenya indicate that TB burden due to COVID-19 related disruptions may be larger than the direct burden of COVID-19 across the continent. The COVID-19 estimates from the WHO regional office for Africa may provide a more realistic account of the counterfactual scenario of widespread community transmission of SARS-CoV-2 infection across the continent. This is because, in contrast to other modelling efforts, the study used contributory factors based on the knowledge of socioecological effects on disease transmission and coronavirus transmission modalities, including gathering, weather, distribution, and sanitation and hygiene practices. These factors, which are not accounted for in other studies that attempted to model the epidemic trajectory and number of deaths under a counterfactual scenario of widespread community transmission of SARS-CoV-2 infection, have been shown to determine the epidemic trajectory of COVID-19 (as indicated above) ${ }^{8-20}$

For malaria, interruption of ITN and SMC coupled with reduction of MCM, which probably reflects a more realistic situation across the continent based on emerging data from the field, would result in 141,000 to 651,000 excess malaria deaths over a 1-year period, depending on the type of COVID-19 response. ${ }^{36}$ In comparison, 82,735 and 189,579 COVID-19 deaths are predicted over 52 weeks of widespread community transmission of SARS- CoV-2 across the WHO African region in the best and worst case scenarios, respectively. ${ }^{9}$

\section{Conclusions}

We found that most studies substantially underestimated the potential implications of COVID-19 on HIV, TB, and malaria in Africa, while overestimating the direct burden of COVID-19 on the continent.

While sparsity of robust prior evidence to guide policy-making in the face of a rapidly growing pandemic without a cure or a vaccine might have warranted the adoption of these COVID-19 control measures adopted across Africa, the data available so far shows that these measures, whose enforcement is typically longer and more stringent across the continent, are not cost effective as the potential adverse 
effects surpass the potential beneficial effects, although the major consequences will be more visible in the mid- and long-term if effective and sustainable measures as discussed here are not put in place. In particular, the COVID-19 emergency measures adopted across Africa have neglected context specific risks and potentials, including the fact that poverty is the main driver or mediator of most public health problems afflicting the continent.

The current analysis, the first of its scope, highlights the difficulties associated with modelling a rapidly evolving pandemic in settings with underlying crises, complex vulnerabilities, and limited data. Overall, availability of quality and detailed patient and epidemiological data is paramount, not only to inform current policies against the ongoing pandemic, but also to improve preparedness for future crises. The effectiveness of many programs and international investment deployed to fight HIV, TB, and malaria across the continent is undermined by chronic social, economic, and political risks. Corruption embedded in health systems across Africa ${ }^{90,91}$ is a major risk for a successful implementation of the solutions proposed here. Therefore, effective social protection, sustainable economic support, and investment in good governance should be part of these efforts, to ensure their resilience to crises such as the COVID-19 pandemic.

\section{Declarations}

\section{Ethics approval and consent to participate}

Ethical approval is not necessary because this research did not collect identifiable human material and data.

\section{Consent for publication}

Not applicable

\section{Availability of data and materials}

The data used in this analysis are drawn from the references provided. 


\section{Competing interests}

None declared.

\section{Funding}

Ministry of Education, Culture, Sports, Science and Technology of Japan.

\section{Authors' contributions}

FA conceived of and designed the research, reviewed the literature, prepared the data, performed the analysis, drafted the manuscript, discussed the results, and contributed to the revision of the final manuscript. BL, AM, and $\mathrm{MH}$ supported data preparation, reviewed the manuscript, supported the interpretation, and contributed to the revision of the final manuscript. All authors read and approved the final manuscript.

\section{Acknowledgements}

Not applicable

\section{Abbreviations}

AIDS acquired immunodeficiency syndrome

ARV antiretroviral drugs

ART antiretroviral therapy

BCG bacillus Calmette-Guérin

COVID-19 coronavirus disease 2019

DALYs disability-adjusted life-years

GATHER Guidelines for Accurate and Transparent Health Estimates Reporting 
LMIC low- and middle-income countries

LTBI latent tuberculosis infection

HIV human immunodeficiency virus

IRS indoor residual spraying

ITN insecticide treated bed nets

IPTp intermittent preventive treatment in pregnancy

IPTi intermittent preventive treatment in infancy

MCM malaria case management

MTCT mother-to-child transmission

PLWHA people living with HIV/AIDS

PRISMA Preferred Reporting Items for Systematic reviews and Meta-Analyses

PMTCT prevention of mother-to-child transmission

SARS-CoV-2 severe acute respiratory syndrome coronavirus 2

SMC seasonal malaria chemoprevention

SSA sub-Saharan Africa

SP sulfadoxine-pyrimethamine

TB tuberculosis

\section{References}

1. Dehning J, Zierenberg J, Spitzner FP, et al. Inferring change points in the spread of COVID-19 reveals the effectiveness of interventions. Science 2020.

2. Islam N, Sharp SJ, Chowell G, et al. Physical distancing interventions and incidence of coronavirus disease 2019: natural experiment in 149 countries. BMJ 2020; 370: $\mathrm{m} 2743$.

3. Flaxman S, Mishra S, Gandy A, et al. Estimating the effects of non-pharmaceutical interventions on COVID-19 in Europe. Nature 2020: 1-8.

4. Hsiang S, Allen D, Annan-Phan S, et al. The effect of large-scale anti-contagion policies on the COVID19 pandemic. Nature 2020; 584(7820): 262-7. 
5. Auger KA, Shah SS, Richardson T, et al. Association between statewide school closure and COVID-19 incidence and mortality in the US. JAMA 2020.

6. Koo JR, Cook AR, Park M, et al. Interventions to mitigate early spread of SARS-CoV-2 in Singapore: a modelling study. The Lancet Infectious Diseases 2020.

7. Chaudhry R, Dranitsaris G, Mubashir T, Bartoszko J, Riazi S. A country level analysis measuring the impact of government actions, country preparedness and socioeconomic factors on COVID-19 mortality and related health outcomes. EClinicalMedicine 2020: 100464.

8. Walker PG, Whittaker C, Watson OJ, et al. The impact of COVID-19 and strategies for mitigation and suppression in low-and middle-income countries. Science 2020.

9. Cabore JW, Karamagi HC, Kipruto H, et al. The potential effects of widespread community transmission of SARS-CoV-2 infection in the World Health Organization African Region: a predictive model. BMJ global health 2020; 5(5): e002647.

10. Dowd JB, Andriano L, Brazel DM, et al. Demographic science aids in understanding the spread and fatality rates of COVID-19. Proceedings of the National Academy of Sciences 2020; 117(18): 9696-8.

11. Esteve A, Permanyer I, Boertien D, Vaupel JW. National age and coresidence patterns shape COVID-19 vulnerability. Proceedings of the National Academy of Sciences 2020; 117(28): 16118-20.

12. Sharma A, Kumar Sharma S, Shi Y, et al. BCG vaccination policy and preventive chloroquine usage: do they have an impact on COVID-19 pandemic? Cell death \& disease 2020; 11(7): 516.

13. Escobar LE, Molina-Cruz A, Barillas-Mury C. BCG vaccine protection from severe coronavirus disease 2019 (COVID-19). Proceedings of the National Academy of Sciences 2020; 117(30): 17720-6.

14. Yitbarek K, Abraham G, Girma T, Tilahun T, Woldie M. The effect of Bacillus Calmette-Guérin (BCG) vaccination in preventing sever infectious respiratory diseases other than TB: Implications for the COVID-19 Pandemic. Vaccine 2020.

15. Diop BZ, Ngom M, Biyong CP, Biyong JNP. The relatively young and rural population may limit the spread and severity of Covid-19 in Africa: a modelling study. BMJ global health 2020; 5(5): e002699.

16. Whittemore PB. COVID-19 fatalities, latitude, sunlight, and vitamin D. American Journal of Infection Control 2020.

17. Sajadi MM, Habibzadeh P, Vintzileos A, Shokouhi S, Miralles-Wilhelm F, Amoroso A. Temperature, Humidity, and Latitude Analysis to Estimate Potential Spread and Seasonality of Coronavirus Disease 2019 (COVID-19). JAMA Netw Open 2020; 3(6): e2011834-e.

18. Miller A, Reandelar MJ, Fasciglione K, Roumenova V, Li Y, Otazu GH. Correlation between universal BCG vaccination policy and reduced morbidity and mortality for COVID-19: an epidemiological study. MedRxiv 2020.

19. Takahashi H. Role of latent tuberculosis infections in reduced COVID-19 mortality: Evidence from an instrumental variable method analysis. Med Hypotheses 2020; 144: 110214-.

20. Clark AJ, Mark; Warren-Gash, Charlotte; Guthrie, Bruce; Wang, Harry H. X.; Mercer, Stewart W.; Sanderson, Colin; McKee, Martin; Troeger, Christopher; Ong, Kanyin L.; Checchi, Francesco; Perel, 
Pablo; Joseph, Sarah; Gibbs, Hamish P.; Banerjee, Amitava; Eggo, Rosalind M. Global, regional, and national estimates of the population at increased risk of severe COVID-19 due to underlying health conditions in 2020: a modelling study. Lancet Glob Health 2020.

21. Elston J, Cartwright C, Ndumbi P, Wright J. The health impact of the 2014-15 Ebola outbreak. Public Health 2017; 143: 60-70.

22. Wilhelm JA, Helleringer S. Utilization of non-Ebola health care services during Ebola outbreaks: a systematic review and meta-analysis. Journal of global health 2019; 9(1).

23. Brolin Ribacke KJ, Saulnier DD, Eriksson A, Von Schreeb J. Effects of the West Africa Ebola virus disease on health-care utilization-a systematic review. Frontiers in public health 2016; 4: 222.

24. Micah AE, Chen CS, Zlavog BS, Hashimi G, Chapin A, Dieleman JL. Trends and drivers of government health spending in sub-Saharan Africa, 1995-2015. BMJ global health 2019; 4(1).

25. Amimo F, Lambert B, Magit A. What does the COVID-19 pandemic mean for HIV, tuberculosis, and malaria control? Tropical Medicine and Health 2020; 48(1): 32.

26. Bell D, Hansen KS, Kiragga AN, Kambugu A, Kissa J, Mbonye AK. Predicting the Impact of COVID-19 and the Potential Impact of the Public Health Response on Disease Burden in Uganda. Am J Trop Med Hyg 2020.

27. Hogan AB, Jewell BL, Sherrard-Smith E, et al. Potential impact of the COVID-19 pandemic on HIV, tuberculosis, and malaria in low-income and middle-income countries: a modelling study. The Lancet Global Health 2020.

28. Jewell BL, Mudimu E, Stover J, et al. Potential effects of disruption to HIV programmes in subSaharan Africa caused by COVID-19: results from multiple mathematical models. The Lancet HIV 2020.

29. Jewell BL, Smith JA, Hallett TB. Understanding the impact of interruptions to HIV services during the COVID-19 pandemic: A modelling study. EClinicalMedicine 2020: 100483.

30. Siedner MJ, Kraemer JD, Meyer MJ, et al. Access to primary healthcare during lockdown measures for COVID-19 in rural South Africa: a longitudinal cohort study. medRxiv 2020.

31. Boulle AD, Mary-Ann; Hussey, Hannah; Ismail, Muzzammil; Morden, Erna; Vundle, Ziyanda; Zweigenthal, Virginia; Mahomed, Hassan; Paleker, Masudah; Pienaar, David; Tembo, Yamanya; Lawrence, Charlene; Isaacs, Washiefa; Mathema, Hlengani; Allen, Derick; Allie, Taryn; Bam, Jamy-Lee; Buddiga, Kasturi; Dane, Pierre; Heekes, Alexa; Matlapeng, Boitumelo; Mutemaringa, Themba; Muzarabani, Luckmore; Phelanyane, Florence; Pienaar, Rory; Rode, Catherine; Smith, Mariette; Tiffin, Nicki; Zinyakatira, Nesbert; Cragg, Carol; Marais, Frederick; Mudaly, Vanessa; Voget, Jacqueline; Davids, Jody; Roodt, Francois; van Zyl Smit, Nellis; Vermeulen, Alda; Adams, Kevin; Audley, Gordon; Bateman, Kathleen; Beckwith, Peter; Bernon, Marc; Blom, Dirk; Boloko, Linda; Botha, Jean; Boutall, Adam; Burmeister, Sean; Cairncross, Lydia; Calligaro, Gregory; Coccia, Cecilia; Corin, Chadwin; Daroowala, Remy; Dave, Joel A.; De Bruyn, Elsa; De Villiers, Martin; Deetlefs, Mimi; Dlamini, Sipho; Du Toit, Thomas; Endres, Wilhelm; Europa, Tarin; Fieggan, Graham; Figaji, Anthony; Frankenfeld, Petro; Gatley, Elizabeth; Gina, Phindile; Govender, Evashan; Grobler, Rochelle; Gule, Manqoba Vusumuzi; 
Hanekom, Christoff; Held, Michael; Heynes, Alana; Hlatswayo, Sabelo; Hodkinson, Bridget; Holtzhausen, Jeanette; Hoosain, Shakeel; Jacobs, Ashely; Kahn, Miriam; Kahn, Thania; Khamajeet, Arvin; Khan, Joubin; Khan, Riaasat; Khwitshana, Alicia; Knight, Lauren; Kooverjee, Sharita; Krogscheepers, Rene; Jacque Kruger, Jean; Kuhn, Suzanne; Laubscher, Kim; Lazarus, John; Le Roux, Jacque; Lee Jones, Scott; Levin, Dion; Maartens, Gary; Majola, Thina; Manganyi, Rodgers; Marais, David; Marais, Suzaan; Maritz, Francois; Maughan, Deborah; Mazondwa, Simthandile; Mbanga, Luyanda; Mbatani, Nomonde; Mbena, Bulewa; Meintjes, Graeme; Mendelson, Marc; Moller, Ernst; Moore, Allison; Ndebele, Babalwa; Nortje, Marc; Ntusi, Ntobeko; Nyengane, Funeka; Ofoegbu, Chima; Papavarnavas, Nectarios; Peter, Jonny; Pickard, Henri; Pluke, Kent; Raubenheimer, Peter J.; Robertson, Gordon; Rozmiarek, Julius; Sayed, A.; Scriba, Matthias; Sekhukhune, Hennie; Singh, Prasun; Smith, Elsabe; Soldati, Vuyolwethu; Stek, Cari; van den Berg, Robert; van der Merwe, Le Roux; Venter, Pieter; Vermooten, Barbra; Viljoen, Gerrit; Viranna, Santhuri; Vogel, Jonno; Vundla, Nokubonga; Wasserman, Sean; Zitha, Eddy; Lomas-Marais, Vanessa; Lombard, Annie; Stuve, Katrin; Viljoen, Werner; Basson, De Vries; Le Roux, Sue; Linden-Mars, Ethel; Victor, Lizanne; Wates, Mark; Zwanepoel, Elbe; Ebrahim, Nabilah; Lahri, Sa'ad; Mnguni, Ayanda; Crede, Thomas; de Man, Martin; Evans, Katya; Hendrikse, Clint; Naude, Jonathan; Parak, Moosa; Szymanski, Patrick; Van Koningsbruggen, Candice; Abrahams, Riezaah; Allwood, Brian; Botha, Christoffel; Henndrik Botha, Matthys; Broadhurst, Alistair; Claasen, Dirkie; Daniel, Che; Dawood, Riyaadh; du Preez, Marie; Du Toit, Nicolene; Erasmus, Kobie; Koegelenberg, Coenraad F. N.; Gabriel, Shiraaz; Hugo, Susan; Jardine, Thabiet; Johannes, Clint; Karamchand, Sumanth; Lalla, Usha; Langenegger, Eduard; Louw, Eize; Mashigo, Boitumelo; Mhlana, Nonte; Mnqwazi, Chizama; Moodley, Ashley; Moodley, Desiree; Moolla, Saadiq; Mowlana, Abdurasiet; Nortje, Andre; Olivier, Elzanne; Parker, Arifa; Paulsen, Chane; Prozesky, Hans; Rood, Jacques; Sabela, Tholakele; Schrueder, Neshaad; Sithole, Nokwanda; Sithole, Sthembiso; Taljaard, Jantjie J.; Titus, Gideon; Van Der Merwe, Tian; van Schalkwyk, Marije; Vazi, Luthando; Viljoen, Abraham J.; Yazied Chothia, Mogamat; Naidoo, Vanessa; Alan Wallis, Lee; Abbass, Mumtaz; Arendse, Juanita; Armien, Rizqa; Bailey, Rochelle; Bello, Muideen; Carelse, Rachel; Forgus, Sheron; Kalawe, Nosi; Kariem, Saadiq; Kotze, Mariska; Lucas, Jonathan; McClaughlin, Juanita; Murie, Kathleen; Najjaar, Leilah; Petersen, Liesel; Porter, James; Shaw, Melanie; Stapar, Dusica; Williams, Michelle; Aldum, Linda; Berkowitz, Natacha; Girran, Raakhee; Lee, Kevin; Naidoo, Lenny; Neumuller, Caroline; Anderson, Kim; Begg, Kerrin; Boerlage, Lisa; Cornell, Morna; de Waal, Renee; Dudley, Lilian; English, Rene; Euvrard, Jonathan; Groenewald, Pam; Jacob, Nisha; Jaspan, Heather; Kalk, Emma; Levitt, Naomi; Malaba, Thoko; Nyakato, Patience; Patten, Gabriela; Schneider, Helen; Shung King, Maylene; Tsondai, Priscilla; Van Duuren, James; van Schaik, Nienke; Blumberg, Lucille; Cohen, Cheryl; Govender, Nelesh; Jassat, Waasila; Kufa, Tendesayi; McCarthy, Kerrigan; Morris, Lynn; Hsiao, Nei-Yuan; Marais, Ruan; Ambler, Jon; Ngwenya, Olina; Osei-Yeboah, Richard; Johnson, Leigh; Kassanjee, Reshma; Tamuhla, Tsaone. Risk factors for COVID-19 death in a population cohort study from the Western Cape Province, South Africa. Clin Infect Dis 2020.

32. Cilloni L, Fu H, Vesga JF, et al. The potential impact of the COVID-19 pandemic on tuberculosis: a modelling analysis. medRxiv 2020. 
33. Glaziou P. Predicted impact of the COVID-19 pandemic on global tuberculosis deaths in 2020. medRxiv 2020.

34. Buonsenso DC, B.; Kallon, M. N.; lodice, F. Child Healthcare and Immunizations in Sub-Saharan Africa During the COVID-19 Pandemic. Front Pediatr 2020; 8.

35. Roberton T, Carter ED, Chou VB, et al. Early estimates of the indirect effects of the COVID-19 pandemic on maternal and child mortality in low-income and middle-income countries: a modelling study. The Lancet Global Health 2020.

36. Sherrard-Smith E, Hogan AB, Hamlet A, et al. The potential public health consequences of COVID-19 on malaria in Africa. Nature medicine 2020: 1-6.

37. World Health Organization. The potential impact of health service disruptions on the burden of malaria: a modelling analysis for countries in sub-Saharan Africa: World Health Organization, 2020.

38. FinMark Trust. Livelihood impacts of Covid-19 in Kenya, Nigeria and South Africa: FinMark Trust, 2020.

39. Human Sciences Research Council. HSRC Study on COVID-19. South Africa: Human Sciences Research Council, 2020.

40. International AIDS Society. Rapid community survey on the impacts of the COVID-19 pandemic outbreak on PLHIV in Zimbabwe: International AIDS Society, 2020.

41. National Institute For Communicable Diseases. Impact of COVID-19 intervention on TB testing in South Africa: National Institute For Communicable Diseases, 2020.

42. The Global Fund. Disruptions in HIV, TB and malaria programs due to COVID-19: The Global Fund, 2020.

43. Davey DLJ, Bekker L-G, Mashele N, Gorbach P, Coates TJ, Myer L. PrEP retention and prescriptions for pregnant women during COVID-19 lockdown in South Africa. The Lancet HIV 2020.

44. Henry R. Innovations in Agriculture and Food Supply in Response to the COVID-19 Pandemic. Mol Plant 2020; 13(8): 1095-7.

45. Teachout M, Zipfel C. The economic impact of COVID-19 lockdowns in sub-Saharan Africa: International Growth Centre, 2020.

46. International Food Policy Research Institute. Poverty and food insecurity could grow dramatically as COVID-19 spreads: International Food Policy Research Institute, 2020.

47. Akiwumi P. COVID-19: A threat to food security in Africa. United Nations Conference on Trade and Deveelopment; 2020: Least Developed Countries and Special Programmes; 2020.

48. United Nations Economic Commission for Africa. ECA estimates billions worth of losses in Africa due to COVID-19 impact: United Nations Economic Commission for Africa, 2020.

49. Chop E, Duggaraju A, Malley A, et al. Food insecurity, sexual risk behavior, and adherence to antiretroviral therapy among women living with HIV: A systematic review. Health care for women international 2017; 38(9): 927-44. 
50. Loevinsohn M. The 2001-03 Famine and the Dynamics of HIV in Malawi: A Natural Experiment. PloS one 2015; 10(9): e0135108-e.

51. Sy KTL, Haw NJL, Uy J. Previous and active tuberculosis increases risk of death and prolongs recovery in patients with COVID-19. Infectious Diseases 2020: 1-6.

52. Vizcarra P, Pérez-Elías MJ, Quereda C, et al. Description of COVID-19 in HIV-infected individuals: a single-centre, prospective cohort. The Lancet HIV 2020.

53. Sendagire $H$, Kaddumukasa $M$, Ndagire $D$, et al. Rapid increase in resistance of Plasmodium falciparum to chloroquine-Fansidar in Uganda and the potential of amodiaquine-Fansidar as a better alternative. Acta Trop 2005; 95(3): 172-82.

54. Alene KA, Wangdi K, Clements ACA. Impact of the COVID-19 Pandemic on Tuberculosis Control: An Overview. Trop Med Infect Dis 2020; 5(3).

55. Singh M, Mynak M, Kumar L, Mathew J, Jindal S. Prevalence and risk factors for transmission of infection among children in household contact with adults having pulmonary tuberculosis. Archives of disease in childhood 2005; 90(6): 624-8.

56. Saunders MJ, Wingfield T, Datta S, et al. A household-level score to predict the risk of tuberculosis among contacts of patients with tuberculosis: a derivation and external validation prospective cohort study. The Lancet Infectious Diseases 2020; 20(1): 110-22.

57. Reeves A, Basu S, McKee M, Sandgren A, Stuckler D, Semenza JC. Tuberculosis control and economic recession: longitudinal study of data from 21 European countries, 1991-2012. Bulletin of the World Health Organization 2015; 93(6): 369-79.

58. Habteyes Hailu T, Azar T, Davoud SHOJAEIZADEH GG. Tuberculosis treatment non-adherence and lost to follow up among TB patients with or without HIV in developing countries: a systematic review. Iranian journal of public health 2015; 44(1): 1.

59. De Schacht C, Mutaquiha C, Faria F, et al. Barriers to access and adherence to tuberculosis services, as perceived by patients: A qualitative study in Mozambique. PloS one 2019; 14(7): e0219470.

60. Daftary A, Padayatchi N, O'Donnell M. Preferential adherence to antiretroviral therapy over tuberculosis treatment: a qualitative study of drug-resistant TB/HIV co-infected patients in South Africa. Global public health 2014; 9(9): 1107-16.

61. O'Donnell MR, Wolf A, Werner L, Horsburgh CR, Padayatchi N. Adherence in the treatment of patients with extensively drug-resistant tuberculosis and HIV in South Africa: a prospective cohort study. Journal of acquired immune deficiency syndromes (1999) 2014; 67(1): 22.

62. Nigusso FT, Mavhandu-Mudzusi AH. Magnitude of non-adherence to antiretroviral therapy and associated factors among adult people living with HIV/AIDS in Benishangul-Gumuz Regional State, Ethiopia. PeerJ 2020; 8: e8558.

63. Weiser SD, Palar K, Frongillo EA, et al. Longitudinal assessment of associations between food insecurity, antiretroviral adherence and HIV treatment outcomes in rural Uganda. AIDS (London, England) 2014; 28(1): 115. 
64. Czaicki NL, Mnyippembe A, Blodgett M, Njau P, McCoy SI. It helps me live, sends my children to school, and feeds me: a qualitative study of how food and cash incentives may improve adherence to treatment and care among adults living with HIV in Tanzania. AIDS care 2017; 29(7): 876-84.

65. Cluver L, Toska E, Orkin F, et al. Achieving equity in HIV-treatment outcomes: can social protection improve adolescent ART-adherence in South Africa? AIDS care 2016; 28(sup2): 73-82.

66. Masa R, Chowa G. A multilevel conceptual framework to understand the role of food insecurity on antiretroviral therapy adherence in low-resource settings: from theory to practice. Social work in public health 2017; 32(5): 324-38.

67. Young S, Wheeler AC, McCoy SI, Weiser SD. A review of the role of food insecurity in adherence to care and treatment among adult and pediatric populations living with HIV and AIDS. AIDS and behavior 2014; 18(5): 505-15.

68. Patel BH, Jeyashree K, Chinnakali P, et al. Cash transfer scheme for people with tuberculosis treated by the National TB Programme in Western India: a mixed methods study. BMJ open 2019; 9 (12): e033158-e.

69. Torrens AW, Rasella D, Boccia D, et al. Effectiveness of a conditional cash transfer programme on TB cure rate: a retrospective cohort study in Brazil. Transactions of the Royal Society of Tropical Medicine and Hygiene 2016; 110(3): 199-206.

70. Reis-Santos B, Shete P, Bertolde A, et al. Tuberculosis in Brazil and cash transfer programs: a longitudinal database study of the effect of cash transfer on cure rates. PloS one 2019; 14(2): e0212617.

71. Carter DJ, Daniel R, Torrens AW, et al. The impact of a cash transfer programme on tuberculosis treatment success rate: a quasi-experimental study in Brazil. BMJ global health 2019; 4(1): e001029.

72. Rai B, Dixit K, Aryal TP, et al. Developing Feasible, Locally Appropriate Socioeconomic Support for TBAffected Households in Nepal. Tropical medicine and infectious disease 2020; 5(2): 98.

73. Lester RT, Ritvo P, Mills EJ, et al. Effects of a mobile phone short message service on antiretroviral treatment adherence in Kenya (WelTel Kenya1): a randomised trial. The Lancet 2010; 376(9755): 1838-45.

74. Nyoni T, Sallah YH, Okumu M, Byansi W, Lipsey K, Small E. The effectiveness of treatment supporter interventions in antiretroviral treatment adherence in sub-Saharan Africa: a systematic review and meta-Analysis. AIDS care 2020; 32(sup2): 214-27.

75. Nakamanya S, Mayanja BN, Muhumuza R, Bukenya D, Seeley J. Are treatment supporters relevant in long-term Antiretroviral Therapy (ART) adherence? Experiences from a long-term ART cohort in Uganda. Global public health 2019; 14(3): 469-80.

76. Nachega JB, Knowlton AR, Deluca A, et al. Treatment Supporter to Improve Adherence to Antiretroviral Therapy in HIV-Infected South African Adults: A Qualitative Study. JAIDS Journal of Acquired Immune Deficiency Syndromes 2006; 43.

77. Kunutsor S, Walley J, Katabira E, et al. Improving Clinic Attendance and Adherence to Antiretroviral Therapy Through a Treatment Supporter Intervention in Uganda: A Randomized Controlled Trial. AIDS 
and behavior 2011; 15(8): 1795-802.

78. Nyoni T, Okumu M. COVID-19-Compliant Strategies for Supporting Treatment Adherence Among People Living with HIV in Sub-Saharan Africa. AIDS and behavior 2020: 1.

79. Kranzer K, Lawn SD, Meyer-Rath G, et al. Feasibility, yield, and cost of active tuberculosis case finding linked to a mobile HIV service in Cape Town, South Africa: a cross-sectional study. PLoS medicine 2012; 9(8): e1001281-e.

80. Labhardt ND, Motlomelo M, Cerutti B, et al. Home-based versus mobile clinic HIV testing and counseling in rural Lesotho: a cluster-randomized trial. PLoS medicine 2014; 11(12): e1001768-e.

81. Babigumira JB, Sethi AK, Smyth KA, Singer ME. Cost effectiveness of facility-based care, homebased care and mobile clinics for provision of antiretroviral therapy in Uganda. Pharmacoeconomics 2009; 27(11): 963-73.

82. Larson B, Schnippel K, Ndibongo B, et al. Rapid point-of-care CD4 testing at mobile HIV testing sites to increase linkage to care: an evaluation of a pilot program in South Africa. Journal of acquired immune deficiency syndromes (1999) 2012; 61(2): e13.

83. Stephanie W, Hill C, Ricks ML, Bennet J, Oriol NE. The scope and impact of mobile health clinics in the United States: a literature review. International journal for equity in health 2017; 16(1): 178.

84. Enthoven AC. Integrated delivery systems: the cure for fragmentation. American Journal of Managed Care 2009; 15(12): S284.

85. Amimo F, Lambert B, Magit A, Sacarlal J, Hashizume M, Shibuya K. Plasmodium falciparum resistance to sulfadoxine-pyrimethamine in Africa: a systematic analysis of national trends. $B M J$ global health 2020.

86. Short R, Gurung R, Rowcliffe M, Hill N, Milner-Gulland EJ. The use of mosquito nets in fisheries: A global perspective. PLOS ONE 2018; 13(1): e0191519.

87. McLean KA, Byanaku A, Kubikonse A, Tshowe V, Katensi S, Lehman AG. Fishing with bed nets on Lake Tanganyika: a randomized survey. Malaria Journal 2014; 13(1): 395.

88. Jones BL, Unsworth RKF. The perverse fisheries consequences of mosquito net malaria prophylaxis in East Africa. Ambio 2020; 49(7): 1257-67.

89. Pearson CA, Jarvis Cl, Davies NG, et al. Projections of COVID-19 epidemics in LMIC countries. 2020.

90. Onwujekwe O, Agwu P, Orjiakor C, et al. Corruption in Anglophone West Africa health systems: a systematic review of its different variants and the factors that sustain them. Health policy and planning 2019; 34(7): 529-43.

91. Hsiao A, Vogt V, Quentin W. Effect of corruption on perceived difficulties in healthcare access in subSaharan Africa. PloS one 2019; 14(8): e0220583.

\section{Figures}




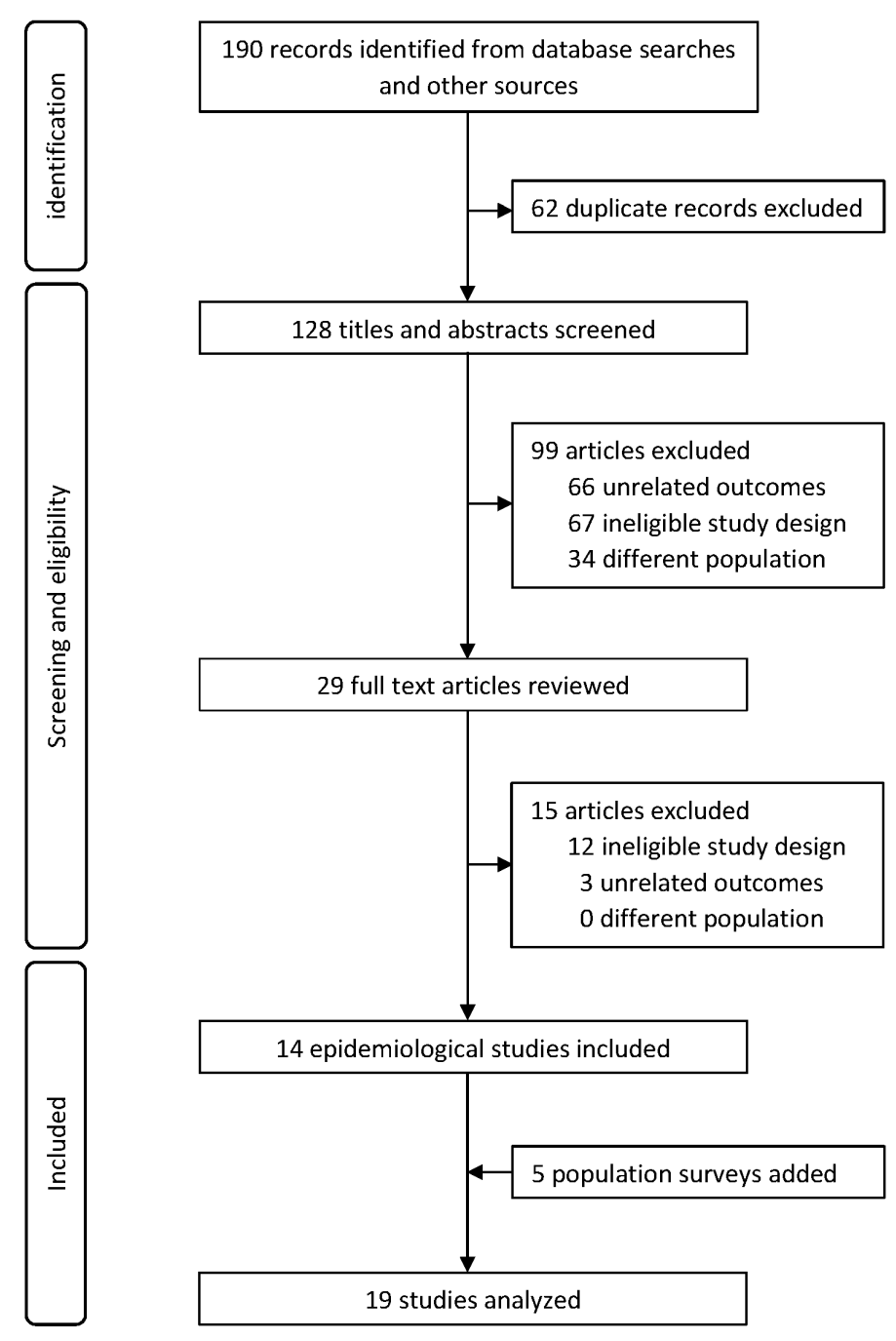

\section{Figure 1}

PRISMA flowchart illustrating eligibility assessment of studies addressing the potential effects of COVID19 on HIV, tuberculosis, or malaria. Our search strategy yielded the following number of records per source: 63 LitCovid, 76 Scopus, 46 MEDLINE (OVID), 8 CINAHL (EBSCO), 22 Web of Science Core Collection, 4 African Index Medicus, 2 Cochrane Library, and 15 other sources. Evidence gathering was done from August 20 to September 15, 2020. The sum of titles and abstracts excluded by each reason > 
total titles and abstracts excluded. This is because titles and abstracts were excluded by not meeting several eligibility criteria.

\section{Supplementary Files}

This is a list of supplementary files associated with this preprint. Click to download.

- Supplement.pdf 